\title{
Monitoring Collaborative Learning Activities: Exploring the Differential Value of Collaborative Flow Patterns for Learning Analytics
}

\author{
María Jesús Rodríguez-Triana*†, Luis P. Prieto*, Alejandra Martínez-Monés ${ }^{\ddagger}$, Juan I. Asensio-Pérez ${ }^{\ddagger}$, \\ and Yannis Dimitriadis ${ }^{\ddagger}$ \\ * Tallinn University, Tallinn, Estonia \\ †École Polytechnique Fédérale Lausanne, Lausanne, Switzerland \\ ¥Universidad de Valladolid, Valladolid, Spain \\ Email: mjrt@tlu.ee, lprisan@tlu.ee, amartine@infor.uva.es, juaase@tel.uva.es, yannis@tel.uva.es
}

\begin{abstract}
Collaborative learning flow patterns (CLFPs) encode solutions to recurrent pedagogical problems, which have been successfully applied to the design of learning experiences. However, the pedagogical knowledge encoded in these patterns has seldom been exploited in learning analytics (LA). This paper analyzes four of the most common CLFPs to extract the intrinsic constraints that lead to a successful collaborative learning activity, and use them to enhance existing LA solutions. To understand the added value of applying such codified knowledge in LA, we present evidence from five authentic case studies in which such constraints aided university teachers in monitoring complex collaborative scripts. The results not only illustrate quantitatively such added value but also unearth qualitative benefits, such as raising practitioners awareness about how the current state of activities may affect future phases of the script.
\end{abstract}

Index Terms-Design patterns; monitoring; learning design; learning analytics; computer-supported collaborative learning.

\section{INTRODUCTION}

Computer-supported collaborative learning (CSCL) poses several orchestration challenges that go from the design and enactment to the assessment of the learning process [1]. In the learning sciences, authors have proposed the use of pedagogical patterns [2] and scripts [3] to help practitioners in the design of learning activities. For instance, in the case of CSCL, collaborative learning flow patterns (CLFPs) guide designers through the application of different learning flow structures that are in accordance with well-known pedagogical theories (e.g., about how to promote effective collaboration), and which have been validated in practice. At the end of this design process, practitioners obtain a CSCL script where the pattern is instantiated in a particular learning context [4].

While patterns and scripts are of great help to conceive the learning activity at design time, further interventions may be required to scaffold interactions towards effective collaboration at learn time [5]. To cope with this problem, the Learning Analytics (LA) community has proposed solutions to support teachers and students in this endeavor, e.g., using monitoring tools that facilitate regulation and guidance [6].

The learning sciences literature has shown that these two kinds of efforts (at design and learn time) can generally be combined, e.g., by monitoring the students' progress according to the pedagogical decisions made at design time [7], [8], and/or notifying teachers about the deviations from the original plan [9]. However, among the studies that illustrate this align- ment between learning design (LD) and learning analytics, research on how the knowledge encoded in the pedagogical patterns impacts the effectiveness of LA solutions is scarce.

In this paper, we focus on the role that learning design patterns (more concretely, CLFPs) may play in the monitoring process of complex collaborative learning activities. First, we analyze four of these patterns (Jigsaw, Pyramid, Role play, and Peer review) extracting their intrinsic constraints, and incorporating them into an LA solution. We then assess the added value that verifying such constraints (i.e., exploiting the knowledge codified in these patterns) has for the performance, novelty and usefulness of the monitoring tool used in five authentic, blended CSCL situations held in university settings.

\section{RELATED WORK}

It has been shown repeatedly in the literature that, when students are asked to collaborate freely, productive interactions do not necessarily occur. Thus, in CSCL, many pedagogical decisions have to do with scaffolding learners towards more effective collaboration [10]. Scripting has proven to be a helpful strategy to promote such effective interactions, by structuring sequences of student actions and activities, to achieve the learning objectives [3].

The conception of CSCL scripts is a complex task, especially for non-expert teachers who may be not used to think in terms of script components (mainly activities, participants, groups, and resources) and mechanisms (such as task distribution among groups and roles, group formation, and sequencing of activities) [10], [11]. To support the script design, the CSCL research community has proposed patterns, such as collaborative learning flow patterns (CLFPs) [4], that reflect good practices in structuring collaborative learning [2], [12].

Depending on the specific context, the design decisions made in relation to the aforementioned components and mechanisms may need adaptation during learn time. Thus, several authors [13]-[15] have analyzed scripts in order to identify which features are modifiable (extrinsic constraints bound to contextual factors) and which ones have to be accomplished in order to keep the essence of the scripts pedagogical intentions (intrinsic constraints bound to the scripts core mechanisms). These intrinsic constraints mainly refer to the group formation policies, the activity flow, and the expected interactions. 
Given the importance of satisfying the CLFP intrinsic constraints in a learning situation, LA solutions could be shaped to monitor them. While some previous studies have focused on how to use CLFP-based scripts to guide the monitoring process [9], [16], in this paper our goal is to extract the CLFPs intrinsic constraints and explore the added value that these constraints may have, when compared with other non CLFPsrelated features of the scripts, for LA solutions that support teachers in monitoring collaborative learning.

\section{CLFP CONSTRAINT ANALYSIS}

CLFPs capture the essence of broadly accepted well-known techniques for structuring the flow of activities that comprise a collaborative learning situation [2], [4], [12]. CLFPs can be used collectively in order to define richer collaborative learning flows, for instance, hierarchically or sequentially, as the studies presented in this paper illustrate.

Based on the description provided by [4] and other related literature, we have identified the (intrinsic) constraints of four of the most commonly used CLFPs (Jigsaw, Pyramid, Peer review, and Role play), which are summarized in Table I. These constraints are related to the script mechanisms and components, and can be classified into three categories: sequence dependencies, group formation policies, and expected interaction (with resources and with peers).

\section{Methodology}

To understand the added value that verifying the pattern constraints had for teachers while monitoring the learning activity, we considered both qualitative (e.g., perceived usefulness) and quantitative data (e.g., accuracy of the LA solution). We illustrate this added value through five case studies conducted in higher education courses, where we explored the following research question: What is the added value of a pattern-aware LA solution for the teacher, versus a pattern-unaware one, or versus the teacher's usual praxis without LA? This question is answered from three perspectives: 1) the role of pattern constraints in the detection of script deviations emerging from the studies (performance), 2) the perceived usefulness, and 3) the novelty of the pattern-aware information for the teacher.

These five studies are part of a long-term design-based research [21] process towards the alignment of scripting and monitoring in blended CSCL scenarios, which took place between 2012 and 2013. We have chosen these studies because they all implemented CLFPs, the teachers had different levels of expertise, but still used the same LA solution. The main characteristics of the studies are summarized in Table II.

We used a mixed methods approach [22], combining multiple informants (i.e., 3 teachers, 1 researcher/observer, 268 students, and the ICT tools used during the study). These informants provided a variety of quantitative and qualitative data, gathered using multiple techniques (analysis of the learning designs, teacher interviews, researcher observations, student questionnaires, system logs, and student-generated artefacts). All these elements allowed for triangulated evidence about the deviations from the script, and their detection by teachers and the LA solution. Besides, the aforementioned qualitative data sources provided evidence about the teachers experience while monitoring the students work.

\section{CAse Studies}

\section{A. Contexts}

The pattern constraints were evaluated in five authentic scenarios with common profile (see Table II): blended CSCL scenarios spanning 3-4 weeks, supported by a virtual learning environment and external web 2.0 tools, with university students. Due to the high risk of failure posed by the interrelated activities, it was crucial for the orchestration to monitor the student activity, assessing how deviations from the plan could impact later activities. Despite these commonalities, each study had a different profile given by the teacher expertise, the number of students, and the implemented CLFPs.

\section{B. Assessing Performance, Novelty and Usefulness}

To measure the added value of taking into consideration the pattern constraints at learn time, we built different versions of the LA solution (different detectors of deviations from the intended script, based on the evidence of student interactions in a distributed learning environment made up of a learning management system and external Web 2.0 tools, see [9]). Along with the teacher-reported usual praxis when monitoring the collaborative activities, we built three models of reality:

1. The teacher model represents the teachers' awareness during the enactment of the scenario, if they had followed their usual praxis (without any LA solution). In our case studies, if teachers had no evidence of a deviation from the script, they assumed everything was going according to the initial plan. To build a detector that modeled the teachers awareness, we asked them about their current awareness of deviations from the script before having access to the LA solution. Since the teachers were the ones designing the learning scenario, they were initially aware of the constraints of the whole learning design (including the ones coming from the CLFPs and the specific activities described in the script).

2. The script-aware model represents teachers awareness if they had used a basic script-aware LA solution. This enables a quantified approximation to the added value of an LA solution that is aware of general script constraints, but ignorant of the pattern constraints (Table I). For example, in CS1, out of 113 constraints, 77 were inferred from the script (e.g., mandatory usage of resources or participation in activities) and 36 from the patterns (Table III, Indicator checks column). Thus, this detector only considered the former subset of constraints.

3 . The pattern-aware model represents the teachers' awareness as they used the pattern-aware LA solution (an extended version of the script-aware one that also included pattern constraints). This detector corresponds to the actual monitoring reports delivered to the teacher during the scenario enactment.

For each detector, we compared the constraints being monitored (Table III, Indicator checks column) with the actual deviations that occurred (i.e., the actual problems extracted 
TABLE I

Overview of the Jigsaw, Pyramid, Peer Review, and Role Play Intrinsic Constraints.

\begin{tabular}{|c|c|}
\hline Jigsaw & Pyramid \\
\hline $\begin{array}{l}\text { - Related works: [4], [15], [17], [18] } \\
\text { - Learning context: Complex problem resolution that can be divided into } \\
\text { sub-problems. } \\
\text { - Learning flow: (1) Each member of a Jigsaw group is assigned a particular } \\
\text { sub-problem. (2) Participants who study the same sub-problem meet in an } \\
\text { Expert group to exchange ideas, becoming experts in the sub problem given } \\
\text { to them. (3) Participants of each Jigsaw group meet to contribute with their } \\
\text { diverse expertise in order to solve the whole problem. } \\
\text { - Constraints: } \\
\text { * There must be at least two subproblems (S) but no more than half the } \\
\text { number of participants }(P): 2 \leq|S| \leq|P| / 2 \\
* \text { There must be at least one expert group }(E) \text { per sub-problem }(S): \forall s \in \\
S, \exists e \in E \\
* \text { The number of jigsaw groups (J) must be proportional to the number of } \\
\text { participants (P) and subproblems (S): }|J|<=|P| /|S| \\
* \text { Each jigsaw group }(J) \text { must have at least one expert (e) per subproblem } \\
\text { (S): } \forall j \in J \wedge \forall s \in S, \exists e \in E \\
* \text { Expert and jigsaw groups }(E, J) \text { must have more than one participant to } \\
\text { enable collaboration: } \forall e \in E,|e|>1 ; \forall j \in J,|j|>1 \\
* \text { The jigsaw group }(J) \text { size must be at least equal to the number of sub- } \\
\text { problems }(S): \forall j \in J,|j| \geq|S| \\
* \text { At least one expert }(e) \text { per sub-problem }(s) \text { must be actively involved from } \\
\text { each jigsaw group }(j): \forall j \in J \wedge \forall s \in S, \exists e \in E\end{array}$ & $\begin{array}{l}\text { - Related works: [4], [13], [19], [20] } \\
\text { - Learning context: Several participants face the resolution of the same } \\
\text { problem. } \\
\text { - Learning flow: (1) Participants (individually or forming an initial small } \\
\text { group) study the problem and propose an initial solution. (2) Groups of } \\
\text { participants compare and discuss their proposals and, finally, propose a new } \\
\text { shared solution. (3) Those groups join in larger groups in order to generate } \\
\text { new agreed proposals. (4) At the end, all the participants must propose a } \\
\text { final and agreed solution. } \\
\text { - Constraints: } \\
\text { * The number of groups between consecutive levels }\left(G_{i}, G_{i-1}\right) \text { must } \\
\text { decrease at least by } 2:\left|G_{i}\right| \leq\left|G_{i-1}\right| / 2 \\
* \text { Except for the first level of the pyramid, each group }(g) \text { must have more } \\
\text { than one participant to enable collaboration: } \forall g \in G_{i}(i>1),|g|>1 \\
* \text { Groups }\left(G_{i}\right) \text { must be formed by at least } 2 \text { groups from the previous level } \\
\text { (Gi-1): } \forall g \in G_{i} \exists g^{\prime}, g^{\prime \prime} \in G_{i-1} / g^{\prime}, g^{\prime \prime} \in g \\
* \text { Groups }\left(G_{i}\right) \text { must have enough active participants }(P) \text { to ensure the } \\
\text { continuity of the next level }\left(G_{i+1}\right): \forall g \in G_{i+1} \exists p_{a} \in g_{i}^{\prime} \wedge p_{b} \in \\
g_{i}^{\prime \prime} / g^{\prime} \wedge g^{\prime \prime} \in G_{i} \\
* \text { Except for the first level of the pyramid, groups }\left(G_{i}\right) \text { must have at least } \\
2 \text { active participants from different groups of the previous level }\left(G_{i-1}\right) \text { : } \\
\forall g \in G_{i}(i>1), \exists\left(p^{\prime}, p^{\prime \prime} \in P\right) \wedge\left(g^{\prime}, g^{\prime \prime} \in G_{i-1}\right) / p^{\prime} \in\left(g \cap g^{\prime}\right) \wedge p^{\prime \prime} \in \\
\left(g \cap g^{\prime \prime}\right)\end{array}$ \\
\hline Peer review & Role Play \\
\hline $\begin{array}{l}\text { - Related works: [4], [13], [19] } \\
\text { - Learning context: Participants with similar knowledge and experience study } \\
\text { a problem. } \\
\text { - Learning flow: (1) Each participant or group develops a solution to a } \\
\text { problem. (2) Reviewers are assigned so that every group reviews at least } \\
\text { one solution and every group receives at least one review. Each reviewer } \\
\text { is given the solution to review, reviews it, and produces feedback for the } \\
\text { author. (3) Groups analyse the received feedback and discuss the feedback } \\
\text { with reviewers. (4) Each groups solution is improved taking into account } \\
\text { reviewers' feedback. } \\
\text { - Constraints: } \\
\text { * There must be at least } 2 \text { groups }(G) \text { to carry out the review: } 2 \leq|G| \geq 2 \\
\text { * There must be at least } 1 \text { participant in each group }(G): \forall g \in G,|g|>1 \\
\text { * Every group }(G) \text { must review at least } 1 \text { solution }(S): \forall g \in G, \exists s \in S \\
* \text { Solutions }(S) \text { must be reviewed by at least } 1 \text { group }(G): \forall s \in S, \exists g \in G\end{array}$ & $\begin{array}{l}\text { - Related works: [4] } \\
\text { - Learning context: Participants play a character in a simulation. } \\
\text { - Learning flow: (1) Participants consult information about the problem to } \\
\text { be simulated and prepare the role of their characters. (2) Participants in the } \\
\text { same simulation group perform a particular situation related to the problem. } \\
\text { (3) Trained simulations are performed to the rest of the class. (4) The whole } \\
\text { class discuss and share their conclusion about the problem. } \\
\text { - Constraints: } \\
\text { * There must be enough groups }(G) \text { to discuss about the simulation: }|G|>1 \\
\text { * Groups' }(G) \text { must have more than one participant to enable collaboration: } \\
\forall g \in G,|g|>1 \\
* \text { In each group }(g) \text {, participants }(P) \text { should have at least one role }(r) \text { : } \\
\forall g \in G a n d \forall p \in P / p \in g, \exists r \in R \\
* \text { In each group }(g), \text { each role }(r) \text { should be taken at by at least one } \\
\text { participant }(p): \forall g \in G a n d \forall r \in R, \exists p \in P / p \in g\end{array}$ \\
\hline
\end{tabular}

TABLE II

Case Study Overview

\begin{tabular}{cccccll}
\hline Study & Teachers & Teacher profile & Students & Duration (weeks) & CLFPs used & Subject \\
\hline CS1 & T1 & Expert & 14 & 3 & Jigsaw, Peer review & Learning methods \\
\hline CS2 & T1 & Expert & 14 & 4 & Pyramid, Peer review & Educational research \\
\hline CS3 & T2 & Non expert & 60 & 3 & Role play, Peer review & Guidance and mentoring for students and families \\
\hline CS3 & T3 & Non expert & 165 & 4 & Jigsaw, Peer review & Psycho-pedagogical basis for attention to diversity \\
\hline CS4 & T1 & Expert & 15 & 3 & Pyramid, Peer review & Educational research \\
\hline
\end{tabular}

from post-hoc questionnaires to students, interviews, observations, and the learning outcomes as reflected in the tools used by students). Comparing the deviations detected (true positives), the false positives, and false negatives of each of these detectors, with the actual deviations that occurred during the enactment, we obtained different performance metrics (presented in Table III). The problem prevalence compares the number of deviations with the total number of indicator checks. The accuracy measures the fraction of all instances that are correctly categorized. Sensitivity (also known as recall or true positive rate) and specificity (or true negative rate) represent respectively the proportion of positives and negatives that are correctly identified as such. The F1 score is the harmonic average of precision (ratio of correctly predicted positive observations to the total positives) and sensitivity.

Additionally, in order to measure the novelty of the information provided by the script-aware and pattern-aware detectors, teachers were asked, before accessing the LA reports, about the deviations that they had already detected by themselves. Once teachers had reviewed the monitoring reports, they specified whether the different results in the LA report were useful or not. The detailed classification of constraints and the 
TABLE III

Performance, Novelty and Usefulness Metrics of the Different Deviation Detectors in the Five Case Studies

\begin{tabular}{|c|c|c|c|c|c|c|c|c|c|c|}
\hline Study & $\begin{array}{c}\text { Deviations } \\
\text { (from script + CLFP) }\end{array}$ & Detector & $\begin{array}{c}\text { Indicator } \\
\text { checks }\end{array}$ & $\begin{array}{c}\text { Problem } \\
\text { prevalence }\end{array}$ & Accuracy & Sensitivity & Specificity & $\begin{array}{c}\text { F1 } \\
\text { score }\end{array}$ & $\begin{array}{l}\text { Novel } \\
\text { results }\end{array}$ & $\begin{array}{l}\text { Useful } \\
\text { results }\end{array}$ \\
\hline \multirow[t]{3}{*}{$\mathrm{CS} 1$} & $16(13+3)$ & Teacher & 113 & 0.14 & 0.93 & 0.50 & 1 & 0.67 & - & - \\
\hline & & Script-aware & 77 & 0.17 & 0.98 & 0.92 & 1 & 0.96 & $37(48 \%)$ & $59(77 \%)$ \\
\hline & & Pattern-aware & 113 & 0.14 & 0.99 & 0.94 & 1 & 0.97 & $64(56 \%)$ & $94(83 \%)$ \\
\hline \multirow[t]{3}{*}{$\mathrm{CS} 2$} & $30(26+4)$ & Teacher & 226 & 0.13 & 0.92 & 0.4 & 1 & - & - & \\
\hline & & Script-aware & 190 & 0.14 & 0.96 & 1 & 0.96 & 0.88 & $75(61 \%)$ & $119(63 \%)$ \\
\hline & & Pattern-aware & 226 & 0.13 & 0.97 & 1 & 0.96 & 0.90 & $137(61 \%)$ & $150(66 \%)$ \\
\hline \multirow[t]{3}{*}{$\mathrm{CS} 3$} & $17(17+0)$ & Teacher & 448 & 0.04 & 0.98 & 0.41 & 1 & 0.58 & - & - \\
\hline & & Script-aware & 368 & 0.05 & 1 & 1 & 1 & 1 & $239(65 \%)$ & $308(84 \%)$ \\
\hline & & Pattern-aware & 448 & 0.04 & 1 & 1 & 1 & 1 & $255(57 \%)$ & $388(87 \%)$ \\
\hline \multirow[t]{3}{*}{ CS4 } & $45(36+9)$ & Teacher & 1217 & 0.04 & 0.97 & 0.15 & 1 & 0.27 & - & - \\
\hline & & Script-aware & 922 & 0.04 & 0.996 & 0.89 & 1 & 0.94 & $904(98 \%)$ & $918(99.6 \%)$ \\
\hline & & Pattern-aware & 1217 & 0.04 & 0.997 & 0.91 & 1 & 0.95 & $1198(98 \%)$ & $1213(99.7 \%)$ \\
\hline \multirow[t]{3}{*}{ CS5 } & $27(27+0)$ & Teacher & 328 & 0.08 & 0.92 & 0 & 1 & NA & - & - \\
\hline & & Script-aware & 280 & 0.10 & 0.97 & 1 & 0.96 & 0.86 & $180(64 \%)$ & $271(96.8 \%)$ \\
\hline & & Pattern-aware & 328 & 0.08 & 0.97 & 1 & 0.97 & 0.86 & $225(69 \%)$ & $319(97.3 \%)$ \\
\hline
\end{tabular}

analysis per case study, along with the $\mathrm{R}$ source code used to analyze the data are also available as additional material ${ }^{1}$.

\section{Findings}

Going back to our research question -What is the added value of a pattern-aware LA solution for the teacher, versus a pattern-unaware one (or script-aware), or versus the teacher's usual praxis without LA?-, this section structures the findings according to the impact of pattern constraints in detecting deviations from the learning design (performance), novelty of the LA-provided information, and usefulness of this information.

Performance. Due to the low frequency of deviations (problem prevalence between 0.04 and 0.17), accuracy values do not differ much (from 0.92 to 0.997 ). Thus, teacher heuristics (assuming that everything went according to the plan in case of no evidence to the contrary) worked well most of the time. However, since teachers could not bear in mind every constraint while monitoring, they often missed the deviations. Therefore, LA solutions led to better overall performance (measured by sensitivity and F1 scores). Nevertheless, the added value of the pattern-aware LA detector (versus the general script-aware one) was not very large, mainly due to a majority of script- (rather than pattern-) related problems.

Novelty. Teachers reported that the LA-provided information was novel quite often $(48 \%-98 \%$ of the pieces of information presented), being the absolute number higher for the pattern-aware than for the script-aware detector $(7-45 \%$ more). While this is an expected result (as more indicator checks are done), also the proportion of novel pieces of information is higher (pattern-related information is most often unknown to the teacher). A potential explanation could be connected to the teachers cognitive load: while the script

\footnotetext{
${ }^{1}$ Additional material: https://github.com/MJRodriguezTriana/ICALT2018 CLFPS_Additional-material
}

constraints are stateless (only affect the current activity), the pattern constraints often have an impact on future activities, and therefore it is more complex to infer their status.

Usefulness. Teachers found the LA-provided information useful in most cases $(63 \%-99.7 \%$ of the information presented), even when it was not novel (i.e., usefulness as confirmatory evidence). The absolute number of useful pieces of information is larger in the pattern-aware than in the scriptaware detector (17-59\% more). Again, while this is expected due to the larger number of indicator checks, in relative numbers, teachers perceived more often as useful the patternrelated pieces of information. It is noteworthy that teachers considered the results useful only if they were accurate.

Finally, it should be noted that novelty and, especially, usefulness are subjective measures, and depend on what each teacher can keep in mind during the enactment, and what kind of things they value more (e.g., novel information vs. true information, deviations vs. evidence of non-deviation, etc.).

\section{CONClusions And Future Work}

This paper provides an analysis of the intrinsic constraints of CLFPs and a first assessment of the added value that including these constraints in an LA system had for teachers, using evidence from five authentic CSCL case studies.

Our comparison of teachers usual monitoring praxis and the use of a script-aware and a pattern-aware LA solutions reveals that, in terms of performance in detecting deviations from the script, the LA solutions obtained substantial gains in terms of sensitivity and F1 scores. However, the low problem prevalence of pattern-related deviations made the improvement due to CLFP-related constraints quite modest (compared to a general script-aware LA solution). Nevertheless, the teachers perceived novelty and usefulness of the information provided were clearly higher in the pattern-aware LA solution since it reveals the impact of the current state on future activities. 
Aside from the usual limitations due to the relatively low number of (authentic) case studies analyzed, the results presented here also are limited to the quantitative evidence from these case studies (due to space limitations). There is, however, additional qualitative evidence supporting the aforementioned results in terms of novelty and usefulness of the information presented to the teachers by the LA solutions.

These results, however, are not to be taken at face value as a quantification of the added value of design patterns for LA. Regardless of the numeric results, our evaluation also unearthed several issues worthy of study and discussion within the CSCL and LA communities, such as: looking beyond plain measures of accuracy and performance, assessing also the subjective aspects of our systems added value (e.g., in terms of novelty, usefulness, workload, ... [23], [24]) and the differential value of different pieces of LA information (e.g., should we emphasize problem detection, and if yes, how much? cf. the well-known negativity bias [25]).

In this paper we also left several avenues of inquiry unexplored, which mark ways forward in our future work on understanding the added value of collaborative pattern knowledge for LA: a) to further analyze the differential value of the different kinds of constraint types (group formation, activity sequence or interaction); b) the exploitation of additional patterns that are recurrent in blended collaborative learning design (e.g., the fact that small-group and individual activities often happen at a distance, while large-group ones are colocated); or c) to apply similar pattern-based approaches to more fine-grained collaborative micro-scripts, probably with the help of machine learning to detect pattern violations in unstructured data (e.g., by analyzing voice or text discourse).

\section{ACKNOWLEDGMENT}

This research has been partially funded by the European Union (grant agreements no. 669074 and 731685), the Spanish Ministries of Economy and Competitiveness (projects TIN2014-53199-C3-2-R and TIN2017-85179-C3-2R) and Science and Education (PRX17/00410), and the Regional Government of Castilla y Len (project VA082U16).

\section{REFERENCES}

[1] P. Dillenbourg, G. Zufferey, H. Alavi, P. Jermann, S. Do-Lenh, Q. Bonnard, S. Cuendet, and F. Kaplan, "Classroom orchestration: The third circle of usability," in International Conference on Computer Supported Collaborative Learning, ser. CSCL'11, 2011, pp. 510-517.

[2] D. Laurillard, Teaching as a Design Science: Building Pedagogical Patterns for Learning and Technology. London, UK: Routledge, 2012.

[3] I. Kollar, F. Fischer, and F. W. Hesse, "Collaboration Scripts - A Conceptual Analysis," Educational Psychology Review, vol. 18, no. 2, pp. 159-185, 2006.

[4] D. Hernández-Leo, J. I. Asensio-Pérez, Y. Dimitriadis, and E. D. Villasclaras-Fernández, "Generating CSCL Scripts: From a Conceptual Model of Pattern Languages to the Design of Real Scripts," in Technology-Enhanced Learning: Design Patterns and Pattern Languages. Rotterdam, Netherlands: Sense Publishers, 2010.

[5] P. Goodyear, "Teaching as design," Herdsa review of higher education, vol. 2, pp. 27-50, 2015.

[6] A. van Leeuwen, J. Janssen, G. Erkens, and M. Brekelmans, "Supporting teachers in guiding collaborating students: Effects of learning analytics in CSCL," Computers \& Education, vol. 79, pp. 28-39, 2014.
[7] L. Lockyer and S. Dawson, "Learning designs and learning analytics," in International Conference on Learning Analytics \& Knowledge, ser. LAK'11. ACM, 2011, pp. 153-156.

[8] A. Martínez-Monés, A. Harrer, and Y. Dimitriadis, "An InteractionAware design process for the integration of interaction analysis into mainstream CSCL practices," in Analyzing Interactions in CSCL. Boston, MA: Springer, 2011, pp. 269-291.

[9] M. J. Rodríguez-Triana, A. Martínez-Monés, J. I. Asensio-Pérez, and Y. Dimitriadis, "Scripting and monitoring meet each other: Aligning learning analytics and learning design to support teachers in orchestrating CSCL situations," British Journal of Educational Technologies, vol. 46, no. 2, pp. 330-343, 2015.

[10] P. Dillenbourg, "Over-scripting CSCL: The risks of blending collaborative learning with instructional design." in Three worlds of CSCL. Can we support CSCL? Heerlen, Nederlands: Open Universiteit Nederland, 2002, pp. 61-91.

[11] P. Häkkinen and K. Mäkitalo-Siegl, "Educational perspectives on scripting CSCL," in Scripting Computer-Supported Collaborative Learning. Boston, MA: Springer, 2007, pp. 263-271.

[12] G. Conole, P. McAndrew, and Y. Dimitriadis, "The role of CSCL pedagogical patterns as mediating artefacts for repurposing Open Educational Resources," in Techniques for Fostering Collaboration in Online Learning Communities: Theoretical and Practical Perspectives. Hershey, USA: IGI Global, 2011, ch. 12.

[13] S. Demetriadis and A. Karakostas, "Adaptive Collaboration Scripting: A Conceptual Framework and a Design Case Study," in International Conference on Complex, Intelligent and Software Intensive Systems, ser. CISIS'08. IEEE Computer Society, 2008, pp. 487-492.

[14] P. Dillenbourg and P. Tchounikine, "Flexibility in macro-scripts for CSCL," Journal of Computer Assisted Learning, vol. 23, no. 1, pp. 1-13, 2007.

[15] M. Pérez-Sanagustín, J. Burgos, D. Hernández-Leo, and J. Blat, "CLFP Intrinsic Constraints-Based Group Management of Blended Learning Situations," in Technology-Enhanced Systems and Tools for Collaborative Learning Scaffolding. Berlin, Heidelberg: Springer, 2011, vol. 350, pp. 115-133.

[16] M. J. Rodríguez-Triana, A. Martínez-Monés, J. I. Asensio-Pérez, I. M. Jorrín-Abellán, and Y. Dimitriadis, "Monitoring pattern-based CSCL scripts: a case study," in European Conference on Technology Enhanced Learning, ser. ECTEL'11, vol. 6964. Springer, 2011, pp. 313-326.

[17] S. Alvino, J. I. AsensioPérez, Y. Dimitriadis, and D. HernándezLeo, "Supporting the reuse of effective CSCL learning designs through social structure representations," Distance Education, vol. 30, no. 2, pp. 239258,2009

[18] M. Balestrini, D. Hernández-Leo, R. Nieves, and J. Blat, "TechnologySupported orchestration matters: Outperforming Paper-Based scripting in a jigsaw classroom," IEEE Transactions on Learning Technologies, vol. 7, no. 1, pp. 17-30, 2014.

[19] A. Karakostas and S. Demetriadis, "Adaptation patterns as a conceptual tool for designing the adaptive operation of CSCL systems," Educational Technology Research and Development, vol. 59, no. 3, pp. 327-349, 2010.

[20] K. Manathunga and D. Hernández-Leo, "Authoring and enactment of mobile pyramid-based collaborative learning activities," British Journal of Educational Technologies, 2017. [Online]. Available: http://dx.doi.org/10.1111/bjet.12588

[21] F. Wang and M. J. Hannafin, "Design-Based Research and TechnologyEnhanced Learning Environments," Educational Technology Research and Development, vol. 53, no. 4, pp. 5-23, 2005.

[22] J. C. Greene, L. Benjamin, and L. Goodyear, "The merits of mixing methods in evaluation," Evaluation, vol. 7, no. 1, pp. 25-44, 2001.

[23] M. Scheffel, H. Drachsler, C. Toisoul, S. Ternier, and M. Specht, "The proof of the pudding: Examining validity and reliability of the evaluation framework for learning analytics," in European Conference on Technology Enhanced Learning. Cham: Springer International Publishing, 2017, pp. 194-208.

[24] M. J. Rodríguez-Triana, L. P. Prieto, A. Martínez-Monés, J. I. AsensioPérez, and Y. Dimitriadis, "The teacher in the loop: Customizing multimodal learning analytics for blended learning," in International Conference on Learning Analytics \& Knowledge, ser. LAK '18. ACM, 2018, pp. 417-426.

[25] A. P. McGraw, J. T. Larsen, D. Kahneman, and D. Schkade, "Comparing gains and losses," Psychological science, vol. 21, no. 10, pp. 1438-1445, 2010. 